lateral nuclei; the product of this gene is proposed to have an analogous function to $\mathrm{I} \kappa \beta^{8-10}$

At present the nature of $\mathrm{IL}-1 \mathrm{R}$ signal

1. Anderson, $K$ V \& Nusslein-Volhard, C. Nature 311 223-227 (1984)

2. Anderson, K. V. \& Nusslein-Volhard, C. in Gametogenesis and the Eariy Embryo (ed. Gall, J.) 177-194 (Liss, New York, 1986)

3. DeLotto, R. \& Spierer, P. Nature 323, 688-692 (1986)

4. Chasan, R. \& Anderson, K. V. Cell 36, 391-400 (1989).

5. Steward, R. Science 238, 692-694 (1987).

6. Ghosh, S. et al Cell 62, 1019-1029 (1990)

7. Steward, R. et al. Cell 55, 487-495 (1988).

7. Steward, R. et al. Cell 55, 487-495 (1988).

9. Roth, S., Stein, D. \& Nusslein-Volhard, C. Cell 59 1189-1202(1989)

10. Steward, R. Cel/59, 1179-1188 (1989).

11. Hashimoto, C., Hudson, K. L. \& Anderson, K. V. Cell 52 269-279 (1988)

12. Keith, F. J. \& Gay, N. J. EMBO J. 9, 4299-4305 (1990)

13. Anderson, K. V. in Genes and Embryos (eds Glover, D. M. \& Hames, B. D.) 1-37 (IRL, Oxford, 1989).

14. Sims, J. E. et al. Proc natn Acad. Sci. U.S.A. 86 8946-8950 (1989)

15. Ghosh, S. \& Baltimore, D. Nature 344, 678-683 (1990) 16. Mizel, S. O'Neill, L. A. Bird T. A. \& Saklatvala, J. Immun. Today 11, 390-394 (1990).

\section{Diffuse bands in emission}

SIR - The origin of the diffuse interstellar absorption bands remains an unsolved problem after more than 50 years of research ${ }^{1,2}$. Here I draw attention to a set of four emission features from the biconical nebula of the Red Rectangle ${ }^{3}$, the wavelengths of which correspond well with those of a recently classified subgroup of diffuse interstellar absorption bands ${ }^{4}$. It is thought that at least one of the diffuse band carriers is formed in the Red Rectangle region.

The diffuse interstellar band spectrum consists of over forty absorption features in the visible spectral region, with a wide variety of intensities and widths ${ }^{1}$. On the basis of the relative strengths of a number of bands along different lines of sight, it has been suggested ${ }^{4}$ that they can be divided into families, one of which includes those bands occurring at wavelengths of $5,797,5,850,6,376,6,379$ and $6,614 \AA$. These bands are reasonably strong and comparatively narrow, their line widths being in the region of $1 \AA$. The Red Rectangle emission spectrum has an unidentified set of broader emission features with peak wavelengths of spatially integrated spectra near $5,799,5,855,6,380$ and 6,615 $\AA$ (ref. 3) and typical widths of about $6 \AA$. These two sets of wavelengths are in remarkably good agreement and the greater widths and small wavelength shifts of the Red Rectangle bands relative to the diffuse interstellar bands can be attributed to the higher temperature close to the star HD44179. Also, the profiles of the bands in absorption (diffuse interstellar bands, DIB) and emission (Red Rectangle, RR) are qualitatively similar; the 5,797 (DIB ${ }^{5}$ ) and 5,799 (RR) profiles have a steep blue side, the $6,379\left(\mathrm{DIB}^{5}\right)$ and 6,380 (RR) bands have approximately symmetrical profiles, and the 6,614 $\left(\mathrm{DIB}^{6}\right.$ ) and 6,615(RR) bands also have a steep blue side. The notable absence transduction is a matter of debate. There is evidence that stimulation of IL-1R causes a rise in cyclic AMP levels and that the cAMPdependent protein kinase $A$ can activate the cytosolic form of NFK $\beta$. Other work suggests that activation involves a GTP-binding protein and a serine-specific protein kinase distinct from protein kinases $\mathrm{A}$ and $\mathrm{C}^{16}$. It has also been suggested that the cytoplasmic domain of IL-1R has a nucleotide binding activity and may act as an integral $G$ protein ${ }^{14}$. In conclusion, the evidence now suggests a hypothesis of dorsal-protein nuclear localization that involves signal transduction by Toll receptor in a pathway similar to that mediated by IL-1R.

\section{Department of Biochemistry,}

Nicholas J. Gay University of Cambridge,

Tennis Court Road,

Cambridge CB2 1QW, UK

of uther diffuse bands from the emission spectrum, such as those at wavelengths of 5,780 and $6,284 \AA$, does not necessarily mean that the carrier is not present; other reasons for non-observation, such as a low fluorescence quantum yield, may prevent detection.

The emission bands of the Red Rectangle are probably due to electronic transitions of one or more gas-phase molecules. The line widths and contours of the corresponding diffuse bands also lie within the known range of spectroscopic characteristics of cold large molecules. Considering briefly the suggestion that the $\mathrm{C}_{60}$ molecule or a derivative might be responsible for the diffuse bands ${ }^{7}$, it is worth noting that the magnitude of the rotational constant is nearly the same whether the $\mathrm{C}_{60}$ entity is neutral or ionized, or even has an included or surface atom. Preliminary simulations of the rotational contour of an electronic transition of a $\mathrm{C}_{60}$ entity at various temperatures show that a small decrease in rotational constant on electronic excitation is sufficient to cause the rotational contour to form a band head (steep blue side) and a red-degraded tail, as observed for the diffuse band/Red Rectangle 5,797/ $5,799-\AA$ and $6,614 / 6,615-\AA$ pairs, thus providing some support for the idea that a $\mathrm{C}_{60}$ entity might be responsible for the common set of bands observed in emission and absorption. Further work is in progress.

Department of Chemistry, P. J. SARRE

University of Nottingham,

University Park,

Nottingham NG7 2RD, UK

1. Herbig, G. H. Astrophys. J. 196, 129-160 (1975)

2. Bromage, G. E. O. Jl. R astron. Soc. 28, 294-297 (1987).

3. Warren-Smith, R. F., Scarrott, S. M. \& Murdin, P. Nature 292 317-319 (1981).

4. Krelowski, J. \& Walker, G. A. H. Astrophys. J. 312, 860 (1987).

Westerlund, B. E. \& Krelowski, J. Astron. Astrophys. 203 134-144 (1988).

6. Herbig, G. H. \& Soderblom, D. R. Astrophys, J. 252, 610 (1982)

Kroto, H. W. Heath, J. R., O'Brien, S. C., Curl, R. F. \& Smaliey, R. E. Nature 318, 162-163 (1985)

\section{Stress and evolution}

SIR - According to Sheldon ${ }^{1}$, gradual phyletic evolution can be sustained only by organisms living in or able to track narrowly fluctuating, slowly changing environments, whereas stasis, almost paradoxically, seems to prevail in widely fluctuating, rapidly changing environments. This generalization, expanded in a recent model $^{2}$, relates in a geological time scale to physical variables such as sea level, substrate and climate. I argue here that it is also consistent with evidence from evolutionary genetics.

Organisms first encountering widely fluctuating, rapidly changing environments would be characterized by high stress levels. Assuming stress to be an environmental factor that causes a potentially injurious change in a biological system ${ }^{3}$, its main impact is at the extreme end of the stress gradient, for example at the boundaries of species, where fitness becomes limiting because of elevated energetic costs. Although geographical limits depend on many different factors, the energetic consequences of climatic extremes are often of major importance ${ }^{3,4}$. The resistance of organisms to a wide range of stresses can be increased by artificial selection, but at the cost of lowered metabolic rate and hence fitness, which would be restrictive for evolutionary change $\mathrm{e}^{4}$

Severe environmental stress increases the phenotypic and genotypic variability of ecologically important traits, including those involved in stress resistance ${ }^{3,4}$. This would be expected at species boundaries, where environmental fluctuations tend to be too severe for adaptation to the stresses. Even with such high variability, the concomitant metabolic and fitness costs would preclude expansions of the species ${ }^{3}$ range into ecologically more extreme habitats, so that rapid evolutionary change would be unlikely. The opposite extreme is an invariant and nonstressful habitat, such as may occur under domestication, where low genetic variability for stress-resistance traits $^{3}$ is restrictive for substantial change despite the availability of sufficient metabolic energy. (In assessing genetic variability, stress-resistance traits are considered directly rather than by the common approach of looking at electrophoretic variants, which cannot provide a complete understanding of traits at the phenotypic level $^{3,4}$.)

Evolutionary change may therefore be most rapid in populations from habitats intermediate between these extremes, where there is some environmental stress associated with sufficient metabolic energy to allow some adaptive change. Furthermore, high phenotypic plasticity is likely in such habitats ${ }^{3}$. Also, ecological systems may be the most diverse under a certain degree of stress $^{5}$, so that the greatest number of species occurs when disturbance is intermediate ${ }^{6}$. These circumstances may be a feature of the 